\title{
The midwives' role in screening for Antenatal Depression (AND) and Postnatal Depression (PND)
}

\author{
Caroline J. Hollins Martin ${ }^{1}$
}

Gail Norris ${ }^{2}$

Colin R. Martin ${ }^{3}$

${ }^{1}$ Caroline J. Hollins Martin, Professor in Maternal Health, School of Health and Social Care, Edinburgh Napier University (ENU), Sighthill Campus, Edinburgh, Scotland, UK, EH11 4BN, Email: c.hollinsmartin@napier.ac.uk

${ }^{2}$ Gail Norris, Senior Lecturer \& Lead Midwife For Education, School of Health and Social Care, Edinburgh Napier University (ENU), Sighthill Campus, Edinburgh, Scotland, UK, EH11 4BN, Email: g.norris@napier.ac.uk

${ }^{3}$ Professor Colin R. Martin, Professor of Perinatal Mental Health, Faculty of Health Sciences, Institute for Clinical and Applied Health Research (ICAHR), Rm 329, Allam Medical Building, University of Hull, Hull, HU6 7RX, UK, Telephone: 01482 463708, Email:

C.R.Martin@hull.ac.uk

\section{Correspondence}

Caroline J. Hollins Martin, Professor in Maternal Health, School of Health and Social Care, Edinburgh Napier University (ENU), Sighthill Campus, Edinburgh, Scotland, UK, EH11 4BN, Email: c.hollinsmartin@napier.ac.uk

This document is the Accepted Manuscript version of a Published Work that appeared in final form in British Journal of Midwifery, copyright $\odot$ MA Healthcare, after peer review and technical editing by the publisher. To access the final edited and published work see https://doi.org/10.12968/bjom.2020.28.9.666. 
The midwives' role in screening for Antenatal Depression (AND) and Postnatal Depression (PND)

\begin{abstract}
This paper is an education paper, which aims to inform midwives of tools available to help them make appropriate provisional diagnosis of perinatal depression. A second aim is to increase midwives' awareness of the relatively newer diagnosis of Antenatal Depression (AND). Of additional clinical importance, is for midwives to recognise that Postnatal Depression (PND) may be a continuation of AND. To date, screening for $A N D$ has received relatively little attention compared with $P N D$, with the evidence-base supporting that the impact can be as severe. It is important for midwives to know that screening for AND can be undertaken using valid and reliable psychometric self-report depression screening questionnaires, which have known validity characteristics and threshold cut-off scores. There are several of these tools available to help midwives make the decision about whether or not to refer the women to the mental health team. Current practice in the UK involves the midwife asking an initial short 2-item 'Whooley Question' screen, which if indicates depression, can be followed up by the women completing a self-report depression screening questionnaire. To highlight their availability, a selection of valid and reliable psychometric self-report depression screening questionnaires are discussed herein, with it being important for midwives to develop a toolkit that can be given to women at clinics, in pamphlets, on-line or embedded into Apps.
\end{abstract}

Key words: antenatal depression, postnatal depression, Edinburgh Postnatal Depression Scale (EPDS), Midwives, Perinatal, Screening 
The midwives' role in screening for Antenatal Depression (AND) and Postnatal Depression (PND)

\section{Key points:}

(1) Midwives screening for Antenatal Depression (AND) is as important as screening for Postnatal Depression (PND).

(2) AND rates are comparable to $P N D$ rates, yet emphasis of perinatal screening is conventionally upon $P N D$.

(3) AND can be successfully and cost-effectively identified by the midwife, through using valid and reliable psychometric self-report depression screening questionnaires, e.g.,

- The 'Whooley Questions'

- Edinburgh Postnatal Depression Scale (EPDS)

- The 9-item Patient Health Questionnaire (PHQ-9)

- Beck Depression Inventory (BDI)

- Hospital Anxiety and Depression Scale (HADS)

- Hamilton Depression Rating Scale (HDRS)

(4) It is important for midwives to have a toolkit that can be given to women at clinics, in pamphlets, on-line or embedded into Apps for self-detection of Perinatal Depression Disorders (PDD).

(5) Further research is needed to examine the degree of overlap of questionnaire items between measures, to determine whether they have acceptable commonality in assessing a consistent conceptual domain of depression. 
The midwives' role in screening for Antenatal Depression (AND) and Postnatal Depression (PND)

\section{Introduction}

Part of the midwives' role is to be vigilant towards detecting mental health problems that childbearing women may already have or develop. Of interest to this paper, is depression experienced across the childbearing spectrum. These are referred to as Perinatal Depression Disorders (PDD) and include Antenatal Depression (AND) and Postnatal Depression (PND). PDD's can have significant consequences for women, partner, families and infant (Rahman et al., 2004), which includes reducing women's social participation, arousing sensitivity towards her new born baby, causing infant malnutrition, physical illness, and subsequent depressive episodes (Dix \& Meuner, 2009; Harvey et al., 2012; Josefsson \& Sydsjö, 2010). To date and in terms of research, $A N D$ has received far less research attention compared with $P N D$, which is noteworthy because:

(1) Rates of $A N D$ and $P N D$ are similar, with some evidence showing that the incidence of $A N D$ may be slightly higher than PND (Gavin et al., 2005).

(2) Evidence supports that AND may be a significant predictor for the woman developing PND (Beck, 2001).

(3) Research based aetiology and interventions are limited for AND, compared with PND (Chojenta et al., 2016; Dennis \& Dowswell, 2013, 2013b; Morrell et al., 2016).

(4) Historical aetiological focus on PND has generally discounted the idea that PND may be an extension of pre-existing AND (Jomeen \& Martin, 2008), yet in some cases this may be the case. 
There is a confusing landscape of $P D D$ 's that can be diagnosed over the antenatal and postnatal period (Jomeen \& Martin, 2008; Martin \& Redshaw, 2018), which is surprising because depression is a commonly observed psychological phenomenon. Yet and of relevance, is that recent studies inform that AND, akin to PND, is associated with adverse perinatal outcomes (Eastwood et al., 2017; Navaratne et al., 2016). In fact, studies have reported a higher prevalence for AND, from 6\% (Eastwood et al., 2017) to 17\% (Leigh \& Milgrom, 2008) and PND, from 6\% to $12 \%$ (Eastwood et al., 2011; Leigh \& Milgrom, 2008). A recent review by Van Neil and Payne (2020) quotes that PDD affects $10-20 \%$ of women during pregnancy and the postpartum period. Also, Sawyer et al. (2010) identified rates of AND as $11.3 \%$ and $P N D$ to be $18.3 \%$.

In comparison, the associated rates of antenatal and postnatal anxiety are $14.8 \%$ and $14 \%$ respectively, with anxiety traditionally thought to co-exist with depression (Christensen et al., 2020; Zigmond \& Snaith, 1983). Additional figures show that rates of severe depression are 3 times higher in the first postnatal year, which is more than at any other time in a woman's life (WHO, 2008). Consequently, it is important for midwives to screen for $P D D$ 's, which across the continuum are labelled as $A N D$ and $P N D$. Also worthy of consideration is whether or not PND is a specific type of depression that has its own distinctive aetiological pathway, or instead is simply a continuation of pre-existing AND.

\section{Aetiological models that underpin Perinatal Depression Disorder (PDD)}

The first aspect of $P D D$ that is important for midwives to acknowledge is that $P N D$ can also profoundly affect husbands and/or partners (Cox, 2005; Psouni et al., 2017; Shaheen et al., 2019), with minimal attention paid to this position. Consequently, it is important for the midwife to have a toolkit of measures they can use to support 
suspicion that $A N D$ or $P N D$ may be present, before referring the woman to a health care professional for formal assessment and diagnosis. Post endorsement of a suspected diagnosis, the midwife can refer the woman or husband/partner for a formal assessment via the 'mental health pathway' outlined by their Health Board (HB). Such action could forestall despair for a couple, with more studies needed to develop and measure effectiveness of different referral systems and treatment programs.

The second aspect of PDD's that requires attention, is the need to understand more about causal factors of $A N D$ and $P N D$. For example, there is persuasive evidence to support that one cause of $P N D$ is routed in biology and hormones (Barry et al., 2015; Mah, 2016; Rogers et al., 2016), with a main contradiction to this argument being that males can also experience PND (Shaheen et al., 2019). The idea that husbands/partners can also exerience $P N D$ is a concept that is antagonistic to biological aetiological models and use of pharmacological interventions to treat $A N D$ and $P N D$ (e.g., selective serotonin reuptake inhibitors) (Ishikawa \& Shiga, 2017; Milgrom et al., 2015; Molyneaux et al., 2018). Conflicting aetiological models about causes of PDD's have created an incomplete picture of cause, effect and appropriate treatments, with more research required in this arena.

A third aspect of $P D D$ 's that requires attention, is that currently the main screening focus of maternity care professionals is upon $P N D$, with it recommended that equal attention be paid to diagnosis and treatment of $A N D$. This recognition in disparity of attention is now just beginning to be acknowledged and yet has to fully infiltrate contemporary clinical guidelines (National Collaborating Centre for Mental Health, 2018). Although $A N D$ is as common as $P N D$, mothers-to-be often miss out on proper treatment due to lack of midwife training. Midwives often do not receive 
sufficient formal training in AND, and because some of the symptoms overlap with minor problems of pregnancy, such as tiredness and emotional instability, AND can be difficult to detect (Jomeen \& Martin, 2008; Jomeen, 2009). Hence and of interest to the midwife, is what screening approach could be used to confirm need for referral of the woman for a formal diagnosis of $A N D$ or $P N D$.

\section{Screening for Perinatal Depression Disorder (PDD)}

At present there is confusion about what self-report measures a midwife could use to initially detect $A N D$ or $P N D$, with most measures validated for use post childbirth. At present in the UK, the Edinburgh Postnatal Depression Scale (EPDS) (Cox et al., 1987 ) is considered the 'gold standard' for initial detection of $P N D$, with it also validated for identifying AND (Murray \& Cox,1990). The midwife should note that cut-off-scores differ between antenatal and postnatal use of the EPDS (discussed later)

(Murray \& Cox, 1990).

It is important that the health care professional uses the same valid and reliable psychometric self-report depression screening questionnaire with the same woman both antenatally and postnatally because:

(1) This provides continuity of assessment, simply because the same questions are being asked at two separate observation points.

(2) It allows a baseline to be recorded in the antenatal period, against which a second observation point can be compared, and improvement or deterioration of symptoms observed.

(3) Consistency in scores across observation points (antenatal \& postnatal), opens a debate about whether AND and PND are discrete conditions or a continuation of the same disorder. 
These 3 points support the inherent fidelity of using the same valid and reliable psychometric self-report depression screening questionnaire before and after birth.

Turning to the content of valid and reliable psychometric self-report depression screening questionnaires. There is an assumption that each scale is measuring the same thing, which raises the concept of whether they are potentially interchangeable (Fried, 2017). It is important to note, that switching scales could introduce sources of error, which is another justification for why the same instrument should be used across longitudinal observation points with the same woman. Midwives can select from a variety of valid and reliable psychometric self-report depression screening questionnaires, which can be used in combination to confirm or eliminate suspicion that a woman may have developed $A N D$ or PND.

\section{(1) The 'Whooley Questions'}

The two 'Whooley Questions' (Whooley et al., 1997) are valid screener items asked by the midwife at the woman's first antenatal 'booking visit' in the UK (McGlone et al., 2016). The 'Whooley Questions' have been shown to have acceptable utility in terms of sensitivity, specificity, and suitability as an initial screen for PDD (Arroll et al., 2005). The 2 'Whooley Questions' follow:

(i) During the past month have you often been bothered by feeling down, depressed, or hopeless?

(ii) During the last month have you often been bothered by having little interest or pleasure in doing things?

The two 'Whooley Questions' originate from the PHQ-9 (Spitzer et al., 1994) and are answered by the woman with a straightforward 'yes' or 'no' response. When a positive screen is found, a third additional question is asked:

(iii) Is this something with which you would like help? 
The National Collaborating Centre for Mental Health (2018) guidelines recommend that the midwife conduct an initial 'Whooley Question' screen, and when depression is indicated the woman is issued with a follow-up valid and reliable psychometric self-report depression screening questionnaire. If total scores suggest that AND may be present, a post-interview assessment is undertaken by a mental health professional and an official diagnosis given. Each HB will have a guideline or protocol outlining their own mental health pathway, which each midwife should be familiar with. Nonetheless, the official screening points may be limited, which supports the idea that midwives pay attention in-between when they are suspicious that a $P D D$ may be present.

\section{(2) The Edinburgh Postnatal Depression Scale (EPDS)}

The EPDS (Cox et al., 1987) is a 10-item easy to administer valid and reliable psychometric self-report depression screening questionnaire. Scores above 9 indicate 'possible depression' and beyond 12 'probable depression'. The EPDS has received many validations of effectiveness at screening for PDD (Hewitt et al., 2010), and has been translated and validated for use in at least 37 languages (Cox et al., 2014). It should be noted that most studies have focused upon use of the EPDS in the postnatal period, with limited research conducted in the antenatal period. One issue that the midwife needs to be aware of, is that EPDS cut-off-scores for case classification differ between the antenatal and postnatal period. Murray and Cox (1990) recommend that a higher threshold for EPDS case classification is used in the antenatal period, compared with the postnatal period (Gibson et al., 2009). For example, the cut-off-score of the original EPDS in the postnatal period is $12 / 13$ (Cox et al., 1987). In comparison, in the antenatal period the cut-off score is $14 / 15$ in the second and third trimesters of pregnancy (Murray \& Cox, 1990; Adewuya et al., 
2006). Currently, there is no universal agreement about one single EPDS threshold cut-off-score for indicating diagnosis of $A N D$ or $P N D$, with this discrepancy causing differences in detection rates. Also, when considering the EPDS for use outside the UK, it is important to note that cut-off-scores for countries and languages differ. To identify these language specific cut-off scores, the midwife can search the data-bases for country specific validation papers. It is important to acknowledge, that substantially more is known about the screening utility of the EPDS, in terms of its psychometric characteristics (i.e., validity, reliability \& factor structure). Validity is largely based upon sensitivity and specificity analysis, which has compared the EPDS against 'gold standards', such as clinical diagnosis according to the National Collaborating Centre for Mental Health (2018). Questions on the EPDS can be viewed at: https://www.fresno.ucsf.edu/pediatrics/downloads/edinburghscale.pdf

\section{(3) The 9-item Patient Health Questionnaire (PHQ-9)}

The 9-item PHQ-9 (Spitzer et al., 1994) can be used to indicate AND or PND using a cut-off-score of (9/10). Please note that the PHQ-9 has different scoring approaches, with it important to be specific about which one should be used in any screening protocol or guideline. In relation to its screening ability, the $P H Q-9$ has similar sensitivity and specificity performance as the EPDS, with this comparable performance suggesting that both instruments are suitable for screening for AND and/or PND (Zhong et al., 2014). Questions on the PHQ-9 can be viewed at: https://www.integration.samhsa.gov/images/res/PHQ\%20-\%20Questions.pdf (4) Beck Depression Inventory-11 (BDI-11)

Three versions of the Beck Depression Inventory (BDI) (Beck et al., 1961) have been developed, with the $B D I-I /$ designed for use with individuals over the age of 13 . The 
$B D I / /$ is a revision of the original $B D I$ (Beck, 1996a), which was adapted in response to the DSM-4 changing its diagnostic criteria for major depressive disorder. The $B D I-/ /$ consists of 21 -items that the woman scores from 0 to 3 , with higher total scores indicating more severe depressive symptoms. Cut-off-scores of the BDI-II rate 0-13 minimal depression, 14-19 mild depression, 20-28 moderate depression, and 29-63 severe depression. Questions on the BDI-11 can be viewed at: https://psychologicalprofessional.com/wp-content/uploads/2017/07/Becks-Depressio n-Inventory-BDI-II.pdf

\section{(5) Hospital Anxiety and Depression Scale (HADS)}

The HADS determines levels of both anxiety and depression, with anxiety considered to co-exist with depression (Zigmond \& Snaith, 1983). The HADS consists of 14-items (7 measure anxiety; 7 measure depression). Items are rated on a 4-point scale from 0 to 3, creating a maximum achievable score of 21 for each subscale (42 whole scale total). Individual sub-scores of $11+$ indicate significant psychiatric comorbidity, scores of 8-10 signify presence of condition, and 7 or less indicate normalcy (Herman, 1997).

There have been many explorations into the HADS measurement characteristics, which have shown the scale to be a reliable and valid screening instrument (Christensen et al., 2020; Martin \& Thompson, 2000; Norton et al., 2013), which is suitable for use across the childbearing spectrum (Jomeen \& Martin, 2004; Karimova \& Martin, 2003; Waqas et al., 2019). Consistent with the EPDS, the HADS has no body related (somatic) items that affect mood, which are caused by physiological changes that occur during the antenatal and postnatal period, (e.g., morning sickness, backache, urinary frequency, anaemia, weight gain, varicosities etc.). Questions on the HADS can be viewed at: https://www.svri.org/sites/default/files/attachments/2016-01-13/HADS.pdf 
(6) Hamilton Depression Rating Scale (HDRS)

The HDRS is a multiple item questionnaire used to indicate depression (Hamilton, 1967). The original 1960 version contains 17-items (HDRS-17), but since then 4 additional questions have been added to provide extra clinical information (Hamilton. 1980). Each item is scored by the respondent on a 3 to 5 point Likert scale, with items asking about mood, feelings of guilt, suicide ideation, insomnia, agitation, anxiety, weight loss and somatic symptoms. Questions on the HADS can be viewed at:

https://dcf.psychiatry.ufl.edu/files/2011/05/HAMILTON-DEPRESSION.pdf Limitations

Having described 6 options that can be used to indicate that a woman has AND or $P N D$, it is important for the midwife to understand limitations of using such valid and reliable psychometric self-report depression screening questionnaires. First, responses can be easily exaggerated or minimized by the person completing items on the scale. Second, the way the midwife administers the scale can impact upon the final total score. Third, when the woman is asked to complete the questionnaire with the midwife present, social expectations may prompt responses that differ to competing the scale in private. Forth, valid and reliable psychometric self-report depression screening questionnaires are usually copywrited, hence only links to the scales have been provided in text for the reader to view question content. Some scales cost money to use, and others are cost free. Check with management whether they already have an annual or ongoing license for use of a chosen scale. Fifth, any valid and reliable psychometric self-report depression screening questionnaire is designed to be a checking device, as opposed to a diagnostic tool. Consequently, any indicative diagnosis of $P D D$ made by the midwife, must be 
followed up with an interview and diagnosis made by a trained mental health professional.

There is also the issue of exchangeability of scales, which involves researchers checking for instrument usefulness by comparing how closely one scale agrees with another. For example, the $B D I-I /$ has been positively correlated with the HDRS (Pearson r of 0.71), which is good agreement (Beck et al., 1996b). In terms of reliability, the $B D I-I /$ has also been shown to have a high one-week test-retest reliability (Pearson $r=0.93$ ), which suggests that the scale is not overly sensitive to daily variations in mood (Beck et al., 1996c). These sorts of inventories have been conducted for all the aforementioned scales, with references available for retrieval in the databases.

\section{Discussion}

Screening with valid and reliable psychometric self-report depression screening questionnaires is fundamentally a cost-effective approach to initially indicate whether a woman has AND or PND. The large amount of choice over which self-report measures a midwife should use is a conundrum, with it noted that apart from the EPDS the other aforementioned scales were developed to detect general depression, as opposed to screening specifically for AND or PND. Clearly, more studies are required to evaluate effectiveness and accuracy of these scales in an antenatal and/or postnatal context. It is also important to identify optimum characteristics of how best to use a single scale or combination of valid and reliable psychometric self-report depression screening questionnaires in a toolkit. Also of importance, is to consistently issue the same scale at set observation points across an individual woman's childbearing spectrum. 
In relation to validation of effectiveness of valid and reliable psychometric self-report depression screening questionnaires, many studies conducted have not been cross-sectional in terms of giving the same scale across set observational timepoints. One study that supports cross-sectional use of the EPDS was conducted by Martin and Redshaw (2018). Although scores changed across two observational time points ( $3 \& 6$ months), the underlying structure of the EPDS was found to be consistent in terms of its psychometric properties (Martin \& Redshaw, 2018).

\section{Selecting an appropriate toolkit}

Conciseness is key when considering which valid and reliable psychometric self-report depression screening questionnaires to include in a toolkit, for the purpose of initially indicating whether or not a woman may have AND or PND. During process of selection for inclusion into clinical protocols and guidelines, it is important to state the cut-off-thresholds for AND or PND. The National Collaborating Centre for Mental Health (2018) states that the main criteria when selecting a valid and reliable psychometric self-report depression screening questionnaire is that the scale should consist of 12-items or less. This restriction is due to time costs and sustaining interest of the woman in terms of full completion. This 12-item restriction promotes that the only suitable scales for use post asking the 2 'Whooley Questions', is the 10-item EPDS or the 9-item $P H Q-9$. This recommended restriction in question numbers is unfortunate, precisely because there are several valid and reliable psychometric self-report depression screening questionnaires that have dependable utility, and which consist of more than the allocated quota of questions (i.e., the 21-item BDI-11; 14-item HADS; 17-item HDRS). Note that the 14-item HADS consists of two 7-item scales (7-items indicate anxiety \& 7-items indicate 
depression), with the 7-items that check for depression possibly being used independently.

In practical terms, these prohibited scales often take similar amount of time for the woman to complete. For instance, the 14-item HADS has had many explorations into its measurement characteristics (Christensen et al., 2020; Martin \& Thompson, 2000; Norton et al, 2013), which includes the context of pregnancy and the postnatal period (Jomeen \& Martin, 2004; Karimova \& Martin, 2003; Waqas et al, 2019). When considering potential utility of the 14-item HADS in a perinatal context, it is helpful to know that it consists of two discrete sub-scales of anxiety (7-items) and depression (7-items). The HADS has 4 more questions than the 10-item EPDS (14 versus 10 respectively). Yet, the advantage the HADS has over the EPDS, is that it screens for both depression and anxiety.

This added screening for anxiety is useful, specifically because anxiety is reported to co-exist with depression (National Collaborating Centre for Mental Health, 2018). In addition to detecting depression, the further benefit of detecting anxiety using the HADS with an additional 4-item effort, is its cost effectiveness in terms of time and resources. Overall and in general terms, the HADS is by far the most frequently used screening measure for identifying both depression and anxiety in a variety of contexts (Christensen et al., 2020). Also, the HADS has a substantial evidence-base to verify its use within a broad range of clinical groups (Christensen et al., 2020; Martin \& Thompson, 2000; Norton, Cosco, Doyle et al., 2013; Jomeen \& Martin, 2004; Karimova \& Martin, 2003; Waqas et al., 2019).

Broader concepts of validity, such as assessing factor structure are generally not considered important when combining self-report measures into toolkits, guidelines and protocols. This oversight brings with it a few challenges worthy of 
consideration. First, the 'Whooley Questions' are valid to use for a quick initial mental health screen. Post assimilating a confirmatory response to the 'Whooley Questions', clinical guidelines usually advise follow-up using either the EPDS or PHQ-9. However, the choice of whether to use either of these self-report measures is arbitrary and rests upon clinician preference.

This quandary about what self-report measures to use to detect $A N D$ and $P N D$, is based upon belief that both the EPDS or $P H Q-9$ are measuring the same, which conceptually makes them essentially interchangeable scales. In relation to this concept of exchangeability, some literature reports that there is little overlap in the content of frequently used valid and reliable psychometric self-report depression screening questionnaires, whilst and in contrast there are reports that exchanging measures produces errors. What this means to the midwife and researchers, is that when comparing across longitudinal observation points over the antenatal and postnatal spectrum, the same valid and reliable psychometric self-report depression screening questionnaire should be used. It is also important that further research in this area of measurement error and exchangeability of valid and reliable psychometric self-report depression screening questionnaires is both required and encouraged.

\section{Conclusion}

Valid and reliable psychometric self-report depression screening questionnaires can be very useful for indicating women who are struggling with depression during the antenatal or postnatal period. Such scales can help midwives screen and refer women for appropriate treatment for their depression from mental health professionals. It is a given, that any screening questionnaire that is embedded into a clinical guideline or protocol should be statistically valid and reliable, with this belief 
based upon a very narrow definition of sensitivity and specificity. This paper has raised important issues for midwives that encompass identification of depression in childbearing women across the perinatal spectrum. What has been identified, is that midwives screening for $A N D$ is as important as screening for $P N D$, simply because rates of both are similar. Prior research emphasis has traditionally been focused upon detecting $P N D$. What is important, is for midwives to develop a toolkit of valid and reliable psychometric self-report depression screening questionnaires that they can use in conjunction with guidelines and protocols. It is also important to make these toolkits available to women free of charge in pamphlets, on-line, and embedded into Apps, so they can self-screen and optionally refer themselves. 


\section{References}

Adewuya A, Ola B, Aloba O (2006) Anxiety disorders among Nigerian women in late pregnancy: a controlled study. Archives Womens Mental Health 9, 325-328.

Arroll B, Goodyear-Smith F, Kerse N, Fishman T, Gunn J (2005) Effect of the addition of a 'help' question to two screening questions on specificity for diagnosis of depression in general practice: diagnostic validity study. British Medical Journal 331, 884.

Barry TJ, Murray L, Fearon RM, Moutsiana C, Cooper P, Goodyer IM, Halligan SL (2015) Maternal postnatal depression predicts altered offspring biological stress reactivity in adulthood. Psychoneuroendocrinology 52, 251-260.

Beck AT, Ward CH, Mendelson M, et al. (1961) An inventory for measuring depression. Archives of General Psychiatry 4, 561-571.

Beck AT, Steer RA, Ball R, Ranieri W (1996a) Comparison of Becks Depression Inventories-1A and 11 in psychiatric outpatients. Journal of Personality Assessment. 67(3), 588-97.

Beck AT, Steer RA, Ball R, Ranieri W (1996b) Comparison of Beck Depression Inventories-1A and 11 in psychiatric outpatients. Journal of Personality Assessment. 67(3), 588-97.

Beck AT, Steer RA and Brown GK (1996c) Manual for the Beck Depression Inventory-II". Psychological Corporation, San Antonio (US).

Beck CT (2001) Predictors of postpartum depression: an update. Nursing Research 50(5), 275-285.

Chojenta CL, Lucke JC, Forder PM, Loxton DJ (2016) Maternal health factors as risks for postnatal depression: a prospective longitudinal study. PloS One 11(1), e0147246.

Christensen AV, Dixon JK, Juel K, Ekholm O, Rasmussen TB, Borregaard B, Berg SK (2020). Psychometric properties of the Danish Hospital Anxiety and Depression Scale in patients with cardiac disease: results from the DenHeart survey. Health Qual Life Outcomes 18(1), 9.

Cox J (2005) Postnatal depression in fathers. Lancet 366(9490), 982.

Cox JL, Holden JM, Sagovsky R (1987) Detection of postnatal depression. Development of the 10-item Edinburgh Postnatal Depression Scale. British Journal of Psychiatry 150, 782-786.

Cox J, Holden J, Henshaw C (2014) Perinatal mental health: the Edinburgh postnatal depression scale (EPDS) manual (2nd edn). RCPsych publications, London (UK). Dennis CL, Dowswell T (2013b) Psychosocial and psychological interventions for preventing postpartum depression. Cochrane Database of Systematic Reviews(2), CD001134. doi:10.1002/14651858.CD001134.pub3 
Dix T, Meunier LN (2009) Depressive symptoms and parenting competence: an analysis of 13 regulatory practices. Developmental Review 29 (Supplement 1), 45-68.

Eastwood J, Ogbo FA, Hendry A, Noble J, Page A, Early Years Research Group (EYRG)(2017) The Impact of Antenatal Depression on Perinatal Outcomes in Australian Women. PLoS One. 12(1), e0169907.

Eastwood JG, Phung H, Barnett B (2011) Postnatal depression and socio-demographic risk: factors associated with Edinburgh depression scale scores in a metropolitan area of new South Wales, Australia. Australian \& New Zealand Journal of Psychiatry. 45(12),1040-1046.

Fried El (2017) The 52 symptoms of major depression: Lack of content overlap among seven common depression scales. Journal of Affective Disorders 208, 191-197.

Gavin NI, Gaynes BN, Lohr KN, Meltzer-Brody S, Gartlehner G, Swinson T (2005) Perinatal depression: a systematic review of prevalence and incidence. Obstetrics and Gynecology 106(5 Pt 1), 1071-1083.

Gibson J, McKenzie-McHarg K, Shakespeare J, Price J, Gray RA (2009) Systematic review of studies validating the Edinburgh Postnatal Depression Scale in antepartum and postpartum women. Acta Psychiatrica Scandinavica 119, 350-364.

Hamilton M (1967) Development of a rating scale for primary depressive illness. British Journal of Social and Clinical Psychology 6, 278-296.

Hamilton M (1980) Rating depressive patients. Journal of Clinical Psychiatry 41, 21-24

Harvey ST, Fisher LJ (2010) Green VM. Evaluating the clinical efficacy of a primary care-focused, nurse-led, consultation liaison model for perinatal mental health. Intern Journal Mental Health Nursing 21(1), 75-81.

Hermann C (1997) International experiences with the Hospital Anxiety and depression Scale. a review of validation data and clinical results. Journal Psychosomatic Research 42, 17-41.

Hewitt CE, Gilbody SM, Mann R, Brealey S (2010) Instruments to identify post-natal depression: which methods have been the most extensively validated, in what setting and in which language? International Journal Psychiatry Clinical Practice 14(1), 72-6.

Ishikawa C, Shiga T (2017) The postnatal 5-HT1A receptor regulates adult anxiety and depression differently via multiple molecules. Progress in

Neuro-Psychopharmacology and Biological Psychiatry, 78, 66-74. 
Jomeen J (2009) Midwives 'need training to spot antenatal depression'. Nursing Times. 7 May 2009. Available at:

https://www.nursingtimes.net/archive/midwives-need-training-to-spot-antenatal-depre ssion-07-05-2009/

Jomeen J, Martin CR (2004) Is the hospital anxiety and depression scale (HADS) a reliable screening tool in early pregnancy? Psychology \& Health 19(6), 787-800.

Jomeen J, Martin CR (2008) Reflections on the notion of post-natal depression following examination of the scoring pattern of women on the EPDS during pregnancy and in the post-natal period. Journal of Psychiatric and Mental Health Nursing 15(8), 645-648.

Josefsson A, Sydsjö G (2007) A follow-up study of postpartum depressed women: recurrent maternal depressive symptoms and child behavior after four years. Archives Women's Mental Health 10, 141-145.

Karimova G, Martin C (2003) A psychometric evaluation of the Hospital Anxiety and Depression Scale during pregnancy. Psychology, Health \& Medicine 8(1), 89-103.

Leigh B, Milgrom J (2008) Factors for antenatal depression, postnatal depression and parenting stress. BMC psychiatry. 8(1),1.

Mah BL (2016) Oxytocin, postnatal depression, and parenting: a systematic review. Harvard Review of Psychiatry 24(1), 1-13.

Martin CR, Redshaw M (2018) Establishing a coherent and replicable measurement model of the Edinburgh Postnatal Depression Scale. Psychiatry Research 264, 182-191.

Martin CR, Thompson DR (2000) A psychometric evaluation of the Hospital Anxiety and Depression Scale in coronary care patients following acute myocardial infarction. Psychology, Health \& Medicine 5(2), 193-201.

McGlone C, Hollins Martin CJ, Furber C (2016) Midwives experiences of asking the Whooley questions to assess current mental health: a qualitative interpretive study. Journal of Reproductive and Infant Psychology 34(4), 383-393.

Milgrom J, Gemmill AW, Ericksen J, Burrows G, Buist A, Reece J (2015) Treatment of postnatal depression with cognitive behavioural therapy, sertraline and combination therapy: a randomised controlled trial. Australian and New Zealand Journal of Psychiatry 49(3), 236-245.

Murray D, Cox JL (1990) Screening for depression during pregnancy with the Edinburgh Depression Scale (EDDS). Journal of Reproductive and Infant Psychology 8, 99-107.

Molyneaux E, Telesia LA, Henshaw C, Boath E, Bradley E, Howard LM (2018) Antidepressants for preventing postnatal depression. Cochrane Database of Systematic Reviews 4, CD004363. 
Morrell CJ, Sutcliffe P, Booth A, Stevens J, Scope A, Stevenson, M, Stewart-Brown $S$ (2016) A systematic review, evidence synthesis and meta-analysis of quantitative and qualitative studies evaluating the clinical effectiveness, the cost-effectiveness, safety and acceptability of interventions to prevent postnatal depression. Health Technology Assessment 20(37), 1-414.

National Collaborating Centre for Mental Health (2018) Antenatal and Postnatal Mental Health. Clinical Management and Service Guidance. Updated edition. National Clinical Guideline Number 192. Available at: https://www.nice.org.uk/guidance/cg192/evidence/full-guideline-pdf-4840896925 National Comorbidity Survey Replication. Archives General Psychiatry 62(6), 593-602.

Navaratne P, Foo XY, Kumar S (2016) Impact of a high Edinburgh postnatal depression scale score on obstetric and perinatal outcomes. International Journal of Scientific Reports. 6, 33544.

Norton S, Cosco T, Doyle F, Done J, Sacker A (2013) The Hospital Anxiety and Depression Scale: a meta confirmatory factor analysis. Journal of Psychosomatic Research 74(1), 74-81.

Psouni E, Agebjorn J, Linder H (2017) Symptoms of depression in Swedish fathers in the postnatal period and development of a screening tool. Scandinavian Journal of Psychology 58(6), 485-496.

Rahman A, lqbal Z, Bunn J et al. (2004) Impact of maternal depression on infant nutritional status and illness: a cohort study. Archives of General Psychiatry 61, 946-52.

Rogers SL, Hughes BA, Tomlinson JW, Blissett J (2016) Cortisol metabolism, postnatal depression and weight changes in the first 12 months postpartum. Clinical Endocrinology 85(6), 881-890.

Sawyer A, Ayers S, Smith H. (2010) Pre and postnatal psychological wellbeing in Africa: a systematic review. Journal Affective Disorders 123, 17-29.

Shaheen NA, AIAtiq Y, Thomas A, Alanazi HA, AlZahrani ZE, Younis SAR, Hussein, MA (2019) Paternal postnatal depression among fathers of newborn in Saudi Arabia. American Journal Mens Health 13(1), 1557988319831219.

Spitzer RL, Williams JB, Kroenke K, Linzer M, deGruy FV, Hahn SR, Johnson JG (1994) Utility of a new procedure for diagnosing mental disorders in primary care. The PRIME-MD 1000 study. JAMA 272(22), 1749-1756.

Van Neil MS, Payne JL (2020) Perinatal depression: a review. Cleveland Clinic Journal of Medicine. 87(5), 273-277. 
Waqas A, Aedma KK, Tariq M, Meraj H, Naveed S (2019) Validity and reliability of the Urdu version of the Hospital Anxiety \& Depression Scale for assessing antenatal anxiety and depression in Pakistan. Asian Journal of Psychiatry 45, 20-25.

Whooley MA, Avins AL, Miranda J, Browner WS (1997) Case-finding instruments for depression. Two questions are as good as many. Journal of General Internal Medicine 12(7), 439-445.

World Health Organization (WHO) 2008) Maternal mental health and child health and development in low and middle income countries: report of the WHOUNFPA meeting. WHO Press, Geneva.

Zhong Q, Gelaye B, Rondon M, Sanchez SE, Garcia PJ, Sanchez E., Williams MA (2014) Comparative performance of Patient Health Questionnaire-9 and Edinburgh Postnatal Depression Scale for screening antepartum depression. Journal of Affective Disorders 162, 1-7.

Zigmond AS, Snaith RP (1983) The hospital anxiety and depression scale. Acta Psychiatrica Scandinavica 67(6), 361-370. 